\title{
A pilot study on the acute effect of orange juice with oat $\beta$-glucan on blood glucose and insulin responses in healthy overweight humans
}

\author{
Svilena Lazarova, Beatriz G. Morillo, Dalia Malkova and Ada L Garcia \\ Human Nutrition, School of Medicine, Dentistry and Nursing, University of Glasgow, Glasgow, UK.
}

Orange juice is a popular drink with high flavanone content linked to protective health effects ${ }^{(1)}$. However, high and regular fruit juice consumption is not recommended because of the negative implications of its high glycaemic load ${ }^{(2)}$. Viscous fibres like oat $\beta$-glucan are effective on reducing glycaemic responses. We hypothesised that adding viscous fibre to orange juice will modulate glycaemia after orange juice consumption $^{(3)}$. The aim of this study was to test the acute effect of combining orange juice with oat $\beta$-glucan on glycaemic responses in overweight subjects.

Using a randomized crossover design, 8 healthy subjects (4 females; 4 males) with a mean BMI of $28.9 \pm 0.8 \mathrm{~kg} / \mathrm{m}^{2}$ and age of 27.4 \pm 0.9 years completed the study. They were allocated to either a control group $(500 \mathrm{ml}$ orange juice with bits $=\mathrm{OJ})$ or an experimental group $(500 \mathrm{ml}$ orange juice with bits with added $3 \mathrm{~g} \beta$-glucan $=\mathrm{OJ}+\mathrm{OB})$ with a minimum of one-week washout period between trials. Participants were instructed to follow a low-polyphenol diet for 2 days before and on the trial day. Fasting baseline blood samples were collected upon arrival at the laboratory followed by ingestion of the experimental beverages. Further blood sampling took place at 15, 30, 60, 120, 180 and 240 min after baseline. A light lunch was provided at 180 min. Glucose levels were measured using the hexokinase method and insulin concentrations were obtained via ELISA kits. Area under the Curve (AUC) was calculated.

Plasma glucose and insulin responses showed peak concentrations in both trials 30 minutes following administration of the test drink, with glucose and insulin levels lower in the OJ+OB condition. After this timepoint, concentrations decreased in both groups but glucose and insulin concentrations remained slightly elevated in OJ+OB compared to OJ until ingestion of the next meal (Figure 1 and 2). Analysis of the time averaged AUC for comparison of the overall effect between the two trials showed lower glucose but higher insulin levels for OJ+OB (not shown).

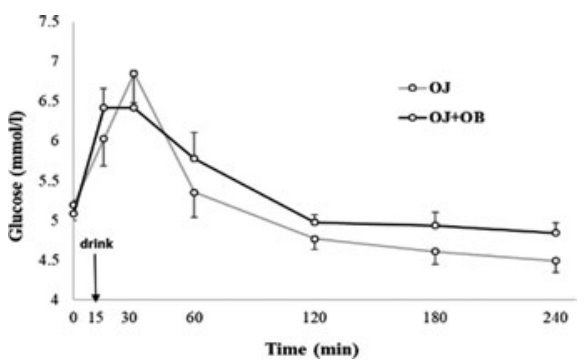

Fig. 1. Plasma glucose responses.

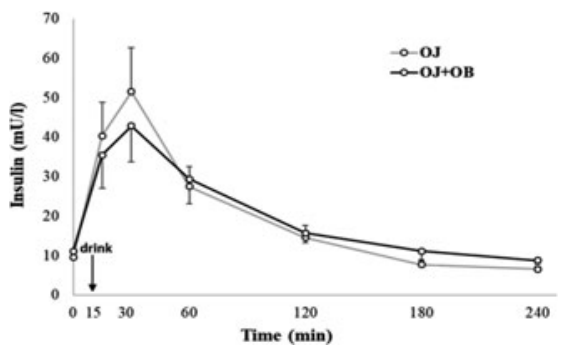

Fig. 2. Plasma insulin responses.

The results from this pilot study point towards a blunted acute glycaemic response when oat $\beta$-glucan is added to orange juice. However, increasing the sample size to reach power $(n=18)$ is needed before conclusive remarks can be made.

1. Khan MK, Dangles O. Journal of Food Composition and Analysis. 2014 Feb 1;33(1):85-104.

2. Basu S, McKee M, Galea G, Stuckler D. American Journal of Public Health. 2013 Nov;103(11):2071-7.

3. Bao L, Cai X, Xu M, Li Y. British Journal of Nutrition. 2014 Aug;112(3):457-66. 